\title{
Microbiota das mãos de mães e de profissionais de saúde de uma maternidade de Goiânia
}

\section{Hand microbiota of mothers and healthcare workers of an obstetric hospital in Goiânia}

\section{Microbiota de las manos de madres y de profesionales de salud de una maternidad en Goiânia}

Marinésia Aparecida Prado Palos ${ }^{\mathrm{I}}$, Dayana Vilas Boas Silva ${ }^{\mathrm{II}}$, Elucir Gir ${ }^{\mathrm{III}}$, Silvia Rita Marin da Silva Canini ${ }^{\mathrm{IV}}$, Patrícia Stanciarini Anders ${ }^{\vee}$, Lara Stefânia Netto de Oliveira LeãoVI , Fabiana Cristina PimentaVII

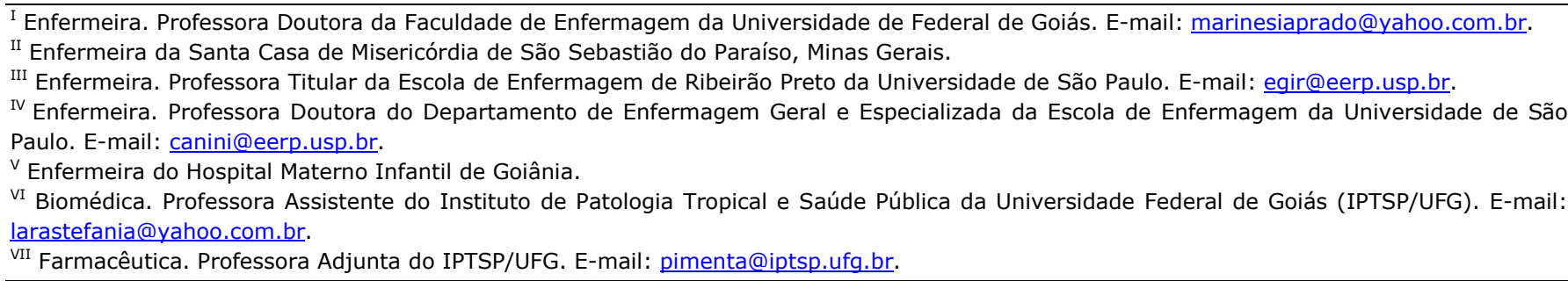

\section{RESUMO}

Trata-se de um estudo descritivo realizado em uma maternidade de um Hospital Universitário de Goiânia-Goiás realizado de abril a outubro de 2003, cujo objetivo foi identificar a microbiota das mãos de mães e de profissionais da área da saúde que cuidam de recém-nascidos. A população do estudo foi composta por todos os trabalhadores da área da saúde que estavam em exercício ativo, e pelas mães que estavam em atendimento no serviço; durante o período do estudo. Das mãos de 31 sujeitos (15 mães e 16 profissionais de saúde) foram isolados cocos Grampositivos, bastonetes Gram-negativos e leveduras, sendo que os microrganismos mais frequentemente isolados foram: Staphylococcus aureus, Staphylococcus e coagulase negativo, Escherichia coli, Klebsiella pneumoniae, Citrobacter freundii, Enterobacter sp, Hafnia alvei, Serratia sp e Arizona sp, os quais têm sido apontados na literatura como associados a surtos de infecção hospitalar em berçários e alojamento conjunto. Assim torna-se premente a elaboração de programas de educação permanente para profissionais e usuários dos serviços de saúde sobre a importância da higienização das mãos, como ferramenta para prevenir infecção e assegurar uma assistência de qualidade aos recém-nascidos.

Descritores: Pessoal de saúde; Infecção hospitalar; Recém-nascido; Lavagem de mãos.

\section{ABSTRACT}

This descriptive study was carried out at a maternity of a University Hospital in Goiânia-Goiás, Brazil, between April and October of 2003. It aimed to identify the hand microbiota of mothers and health professionals delivering care to newborns. The study population consisted of all active health workers and the mothers receiving care at the service during the study period. From the hands of 31 subjects (15 mothers and 16 health professionals), Gram-positive organisms, Gram-negative rods and yeasts were isolated. The most frequently isolated microorganisms were: Staphylococcus aureus, Staphylococcus e negative-coagulase, Escherichia coli, Klebsiella pneumoniae, Citrobacter freundii, Enterobacter sp, Hafnia alvei, Serratia sp and Arizona sp, which literature has appointed as being associated with outbreaks of hospital infection in child-care and rooming-in units. This reveals the urgent need to elaborate permanent education programs for professionals and health service users about the importance of hand washing as a tool to prevent infection and guarantee quality care to newborns.

Descriptors: Health personnel; Cross infection; Newborn,infant; Handwashing.

\section{RESUMEN}

Se trata de un estudio descriptivo realizado en una maternidad de un Hospital Universitario de Goiânia-Goiás, Brasil, entre abril y octubre de 2003, con objeto de identificar la microbiota de las manos de madres y de profesionales del área de la salud que cuidan de recién-nacidos. La población del estudio fue compuesta por todos los trabajadores del área de la salud que estaban en ejercicio activo, y por las madres que estaban recibiendo atención en el servicio durante el período del estudio. De las manos de 31 sujetos (15 madres y 16 profesionales de salud) fueron aislados cocos Gram-positivos, bastones Gram-negativos y levaduras, siendo que los microorganismos más frecuentemente aislados fueron: Staphylococcus aureus, Staphylococcus e coagulase negativo, Escherichia coli, Klebsiella pneumoniae, Citrobacter freundii, Enterobacter sp, Hafnia alvei, Serratia sp e Arizona sp, los cuales han sido indicados en la literatura como asociados a irrupciones de infección hospitalaria en nidos y alojamiento conjunto. Así, se vuelve urgente la elaboración de programas de educación permanente para profesionales y usuarios de los servicios de salud sobre la importancia de la higienización de las manos, como herramienta para prevenir infección y garantizar una atención de calidad a los recién-nacidos.

Descriptores: Personal de salud; Infección hospitalaria; Recién-nascido; Lavado de manos. 


\section{NTRODUÇÃO}

As recomendações de Semmelweis e de Florence Nightingale sobre as medidas de higiene das mãos tornaram-se tão importantes que são lembradas até hoje. Embora seja uma medida de eficácia comprovada, sua operacionalização nos serviços de saúde é complexa e difícil ${ }^{(1)}$.

Apesar de a higienização das mãos ser considerada a principal medida para o controle de infecção hospitalar, ainda hoje os profissionais da área da saúde não a incorporaram totalmente a suas atividades diárias, sendo que apenas $40 \%$ dos profissionais da área da saúde realizam este procedimento $^{(2)}$. A baixa adesão pode ser influenciada tanto por fatores individuais como institucionais, assim cabe às instituições fornecerem condições adequadas para a higienização das mãos.

A pele é colonizada por uma microbiota autóctone estável e cada região anatômica como, por exemplo, a boca, o nariz e o períneo possuem uma microbiota específica. Os microrganismos que compõem essa microbiota são de baixa virulência, encontram-se nas camadas mais profundas podendo se multiplicar na pele e embora em menor freqüência podem ser responsáveis por infecções sistêmicas graves, principalmente em pacientes debilitados e por ocasião da realização de procedimentos invasivos, sendo que para inativá-la é necessária a aplicação de anti-sépticos ${ }^{(3)}$.

A microbiota transitória se encontra na superfície da pele, é facilmente removida necessitando apenas de água e sabão. Estes microrganismos sobrevivem aproximadamente por 48 horas, e são considerados os principais responsáveis pelas infecções hospitalares ${ }^{(3)}$ : 1 -cocos Gram-positivos: Staphylococcus aureus meticilina resistente (MRSA), Staphylococcus coagulase negativo,e Enterococcus faecalis e Enterococcus faecium; 2- bastonetes Gram-negativos: Escherichia coli, Klebsiella pneumoniae, Enterobacter sp, Proteus mirabilis, Citrobacter sp, bastonetes Gram- negativos (BGN) produtores de beta-lactamase de expectro ampliado (BLEA); 3-bastonetes Gram-negativo não fermentadores (BGNNF): Pseudomonas aeruginosa, Burkolderia cepacia, Acinobacter baumanii, Stenotrophomonas maltophilia, entre outros.

Assim, a simples higienização das mãos com água e sabão(1) é suficiente para a remoção da sujidade e remoção da microbiota transitória e consequentemente diminuição da incidência de infecção hospitalar.

Particularmente, nas maternidades, os profissionais de saúde e as mães manuseiam o recém-nascido diariamente, devendo-se considerar que estes são desprovidos de imunidade e de microbiota própria, condição que os colocam mais vulneráveis às infecções hospitalares, reforçando a importância de os profissionais e das mães manterem suas mãos higienizadas. Essa situação despertou-nos para a necessidade de realizar um estudo com o objetivo de identificar os microrganismos das mãos de mães e de profissionais da saúde responsáveis pela assistência ao recém-nascido.

\section{METODOLOGI A}

Trata-se de um estudo descritivo de caráter prospectivo, realizado numa maternidade de um Hospital Universitário de Goiânia-Goiás, no período de abril a outubro de 2003.

As amostras foram coletadas após o parecer favorável do Comitê de Ética e Pesquisa da referida instituição, Protocolo CEPMHA/SCMG 010/2003 e a assinatura do Termo de Consentimento e Livre Esclarecido pelos sujeitos. Médicos, enfermeiros, técnicos e auxiliares de enfermagem, acadêmicos de enfermagem e de medicina e mães realizaram a higiene das mãos, por um minuto, utilizando $10 \mathrm{ml}$ de caldo infusão de cérebro e coração vertido em sacos plásticos estéreis. Em seguida estes foram fechados, acondicionados e transportados imediatamente ao Laboratório de Bacteriologia do Instituto de Patologia Tropical e Saúde Pública da Universidade de Goiás. Posteriormente, $20 \mu \mathrm{l}$ de cada amostra foi semeado em ágar manitol, ágar MacConkey e ágar Sabouraud. As colônias desenvolvidas foram contadas e submetidas à fenotipagem. As leveduras e fungos filamentosos foram caracterizados pela morfologia macroscópica e coloração de Gram. Os resultados foram armazenados e processados por meio de estatística descritiva, utilizando-se programa Epi-Info (versão 6.0-Center for Disease Control).

\section{RESULTADOS E DISCUSSÃO}

Participaram do estudo 31 sujeitos, sendo 16 $(51,6 \%)$ profissionais de saúde e $15(48,4 \%)$ mães de recém-nascidos que prestavam cuidados diretos aos recém-nascidos.

Os profissionais de saúde pertenciam a uma faixa etária entre 22 e 57 anos de idade, 93,8\% eram do sexo feminino e $6,2 \%$ do sexo masculino. Quanto à categoria profissional $6,2 \%$ eram médicos, $18,7 \%$ enfermeiros, $43,6 \%$ técnicos de enfermagem, $19,1 \%$ auxiliares de enfermagem, 6,2\% acadêmicos de enfermagem e 6,2\% acadêmicos de medicina.

Em relação à carga horária semanal de trabalho realizada pelos profissionais variou de 30 a 64 horas por semana, sendo que $43,8 \%$ cumpriam carga horária de 30 horas semanais. O tempo de serviço variou de três meses a quarenta e um anos de trabalho, com média de 10 anos. Ressalta-se que dos 16 profissionais de saúde participantes do estudo 06 $(37,5 \%)$ tinham um segundo vínculo de trabalho.

O número de unidades formadoras de colônias (ufc), dos microrganismos isolados das mãos das 
mães e dos profissionais de saúde, variou de 20 a 7.600.000ufc/ml.

A condição para que o profissional fosse considerado colonizado foi a partir de $20 \mathrm{ufc} / \mathrm{mL}$ de solução de caldo, uma vez que até o momento, esse padrão não está definido na literatura. A dose infectante (número de $\mathrm{ufc} / \mathrm{mL}$ ) para a maioria dos microrganismos capaz de induzir infecção nas pessoas, no ambiente domiciliar e nosocomial, ainda não está estabelecida(4), pois diferentes fatores estão envolvidos no desencadeamento dos casos de infecção nosocomial. Quanto maior a concentração de unidades formadoras de colônias, maior o risco de contaminação e eliciação da infecção.

Dentre os microrganismos isolados destacaramse os cocos Gram-positivos nas mãos de $87,5 \%$ dos profissionais de saúde e $93,3 \%$ das mães; os bastonetes Gram- negativos em 50,0\% das mãos de profissionais e $60,0 \%$ das mães; leveduras/fungos em $93,7 \%$ dos profissionais de saúde e $100 \%$ das mães, conforme observado na Tabela 1 .

Tabela 1: Distribuição dos profissionais de saúde e de mães de recém-nascidos $(N=31)$, segundo o tipo de microrganismo isolado, Goiânia/GO, 2003

\begin{tabular}{|c|c|c|c|c|c|c|}
\hline \multirow{2}{*}{ Tipo de microrganismo } & \multicolumn{2}{|c|}{$\begin{array}{l}\text { Profissional de saúde } \\
\qquad(\mathrm{N}=16)\end{array}$} & \multicolumn{2}{|c|}{$\begin{array}{l}\text { Mães de recém-nascidos } \\
\qquad(\mathrm{N}=15)\end{array}$} & \multicolumn{2}{|c|}{ Total } \\
\hline & $\mathbf{N}$ & $\%$ & $\mathbf{N}$ & $\%$ & $\mathbf{N}$ & $\%$ \\
\hline Cocos Gram positivos & 14 & 87,5 & 14 & 93,3 & 28 & 90,3 \\
\hline Staphylococcus aureus & 10 & 62,5 & 11 & 73,3 & 21 & 67,7 \\
\hline Estafilococos coagulase-negativo & 04 & 25,0 & 10 & 66,6 & 14 & 45,2 \\
\hline Bastonetes Gram negativos & 08 & 50,0 & 09 & 60,0 & 17 & 54,8 \\
\hline Escherichia coli & 00 & 0,0 & 01 & 6,7 & 01 & 3,2 \\
\hline Hafnia alvei & 01 & 6,3 & 02 & 13,3 & 03 & 9,7 \\
\hline Klebsiella pneumoniae & 01 & 6,3 & 00 & 0,0 & 01 & 3,2 \\
\hline Citrobacter freundii & 01 & 6,3 & 00 & 0,0 & 01 & 3,2 \\
\hline Enterobacter $\mathrm{sp}$ & 03 & 18,7 & 03 & 20,0 & 06 & 19,3 \\
\hline Serratia sp & 05 & 31,2 & 01 & 13,0 & 07 & 22,6 \\
\hline Arizona sp & 00 & 0,0 & 13 & 86,7 & 24 & 77,4 \\
\hline Leveduras & 11 & 68,7 & 13 & $\mathbf{8 6 , 7}$ & 24 & 77,4 \\
\hline Fungos filamentosos & 04 & 25,0 & 02 & 13,3 & 06 & 19,3 \\
\hline
\end{tabular}

Dentre os microrganismos isolados das mãos de profissionais e de mães, predominou os cocos Gram positivos: Staphylococcus aureus, Staphylococcus coagulase negativo (Tabela 1). Esses microrganismos, em particular o Staphylococcus aureus, é considerado um dos principais agentes de infecção hospitalar, para os quais já se enfrenta grande dificuldade de obterem-se antimicrobianos ativos para o seu controle. Sob tal ótica, prevê-se potencial de risco para a emergência de futura catástrofe no campo da saúde pública mundial ${ }^{(5-6)}$.

Costa et al $^{(7)}$ identificaram Staphylococcus $\mathrm{sp}$. em mãos e sob as unhas de profissionais de saúde, estudantes e visitantes em ambiente hospitalar, revelando que mais de $50 \%$ estavam colonizados, sendo $7 \%$ por Staphylococcus aureus e um (1/180) profissional estava colonizado por Staphylococcus aureus resistentes à meticilina. Estudo sobre a prevalência de Staphylococcus aureus e de enterobactérias resistentes, em RN de baixo peso, em um berçário de alto risco de um Hospital Escola constatou que $70,0 \%$ dos casos de infecção eram de origem hospitalar ${ }^{(8)}$.

Dentre os bastonetes Gram-negativos identificou-se Escherichia coli, Klebsiella pneumoniae, Citrobacter freundii, Enterobacter sp., Hafnia alvei,
Serratia sp e Arizona sp., como também leveduras e fungos (Tabela 1).

Vários microrganismos estiveram associados à infecção hospitalar em recém nascidos que foram a óbito em uma UTI neonatal, como o Enterobacter sp. $(18,5 \%)$, Staphylococcus aureus (15,7\%), Staphylococcus epidermidis $(11,7 \%)$, Klebsiella pneumoniae $(10,5 \%)$ e Candida sp. (3,4\%). Dentre os sítios mais acometidos destacaram-se a corrente sanguínea $(43,5 \%)$, o globo ocular (13\%) e o tegumento $(9,6 \%)^{(8-9)}$. No presente estudo foram detectados nas mãos dos participantes microrganismos das mesmas espécies.

Em 1999, pesquisadores acompanharam os casos de infecção hospitalar durante sete meses em uma UTI neonatal de um Hospital Escola de Taiwan $^{(10)}$ e detectaram 09 casos de infecção por Serratia marcescens. Destes, 04 apresentaram bacteremia, 03 pneumonia, 01 conjuntivite e 01 infecção de cordão umbilical. Este microrganismo foi mais isolado no sangue (04), seguido por secreção da orofaringe (03), do cordão umbilical (01) e do olho (01). A mesma bactéria também foi isolada nas pias, portas das incubadoras, nas torneiras, no dosador de anti-séptico e nas mãos dos profissionais.

Investigações $^{(11-12)}$ de surtos de Pseudomonas aeruginosas, em unidades de terapia intensiva 
neonatal, comprovaram que os microrganismos foram veiculados por meio das mãos dos profissionais de saúde, as quais foram contaminadas por antisépticos utilizados para a higienização das mesmas e que 06 crianças evoluíram para septicemia e consequentemente foram a óbito.

Estudo realizado com o objetivo de determinar os microrganismos mais freqüentes em unidade de cuidado intensivo neonatal, por um período de seis meses, identificou que 54 recém-nascidos foram acometidos por algum tipo de infecção durante a internação. Foram isolados 144 patógenos sendo que $80 \%$ eram bastonetes Gram-negativas e as mais frequentes foram Acinetobacter spp., Pseudomonas spp. e Klebsiella/Enterobacter spp. Dentre as bactérias Gram-positivas, os mais prevalentes foram os Staphylococcus coagulase-negativo e o Staphylococcus aureus. Todos os microrganismos isolados mostraram-se resistentes a maioria dos antimicrobianos utilizados na referida instituição. E os autores concluíram que o controle ambiental em torno dos neonatos e a política de antibióticos estrita, acrescidas da higienização de mãos, são medidas importantes na prevenção da transmissão nosocomial das bactérias resistentes nestas unidades ${ }^{(12)}$.

Em se tratando de fungos, a colonização por Candida spp é considerada um dos principais fatores de risco para o desenvolvimento do sepsis fúngica em neonatos, mas pouco sabemos sobre as variáveis associadas com a progressão da doença invasiva em neonatos já colonizados. Segundo estudo que analisou 201 neonatos, dos quais 31,1\% eram de baixo peso, a infecção fúngica ocorreu com 51 $(8,1 \%)$ deles. O uso de cateter venoso central, de tubo endotraqueal e a aspiração gástrica foram associados com os casos de sepsis fúngica, e a profilaxia com fluconazol foi associada a um risco diminuído de septicemia nesta população(13).

A higiene das mãos é considerada a medida mais eficaz para impedir a transmissão cruzada de patógenos causadores de infecções, associadas ao cuidado em saúde. Sendo assim, em outubro 2005, a Organização Mundial de Saúde (OMS) lançou o primeiro desafio "Paciente global 2005-2006" com o objetivo de promover maior segurança para o paciente, cujo tema é "cuidado limpo é um cuidado mais seguro", e também propôs explorar o comportamento subjacente dos profissionais da saúde e os aspectos relacionados à adesão a higienização das mãos, bem como elaborar novos Guidelines acerca da higiene das mãos ${ }^{(14)}$.

Compreender como a valorização, ou não, das medidas preventivas em saúde ocorre, pode contribuir para a elaboração de estratégias inovadoras na educação permanente dos profissionais da área da saúde, tendo como alvo principal o próprio profissional ${ }^{(15)}$.
A higienização das mãos é recomendada antes de calçar as luvas, imediatamente após a sua remoção e antes da realização de qualquer procedimento. A utilização de água e sabão ou antiséptico, além de produzir uma limpeza eficiente, remove a microbiota transitória. Porém, não há evidência epidemiológica de que o uso de produtos anti-sépticos ofereça benefícios adicionais na prevenção e transmissão de patógenos ${ }^{(16)}$.

Estudo(17) $^{(17}$ que observou a freqüência da higienização das mãos da equipe multiprofissional, de uma Unidade de Terapia Intensiva, revelou que era esperado que 23 profissionais da equipe de enfermagem realizassem a higienização das mãos 298 vezes, entretanto, a adesão desses profissionais foi de apenas 45/298 (15,0\%). Já para 06 médicos, era esperado que as sua mãos fossem higienizadas 34 vezes, porém esse procedimento ocorreu 9/34 $(26,4 \%)$; com relação aos 02 técnicos de laboratório e a 01 fisioterapeuta, o número previsto foi de 25, entretanto, a higienização das mãos foi realizada uma única vez.

Em ambientes onde os profissionais têm contato freqüente com grande número de pacientes e tempo escasso, o uso de soluções alcoólicas glicerinadas, lenços de base alcoólica, álcool gel, espumas e outros produtos estão recomendado como complemento à higienização das mãos, mas não como substituto ${ }^{(2)}$.

Entretanto, diversos fatores podem interferir na eficácia dessas soluções sobre os microrganismos, como por exemplo, a aplicação de quantidade insuficiente dos produtos nas mãos e ou presença de sujidade nas mãos. A instrução dos profissionais de saúde acerca da técnica apropriada para o uso de anti-séptico pode aumentar significativamente a sua ação sobre as bactérias, desde que a fricção possa abranger todas as regiões das mãos, por um período de um minuto e deixando-as secar naturalmente ${ }^{(18)}$.

Apesar de ser bastante prática, esta opção não deve ser utilizada em detrimento da higienização das mãos com água e sabão, que é considerada a medida mais importante no controle das infecções hospitalares ${ }^{(19)}$.

A higiene das mãos deve ser parte de uma aproximação integrada ao controle da infecção. As Comissões de Controle de Infecção Hospitalar devem ter como estratégia primordial a capacidade de influenciar a mudança de comportamento das pessoas no cotidiano de seu trabalho, bem como aplicar medidas eficazes de controle das infecções, sendo a higienização das mãos uma das medidas mais relevantes.

Nas unidades neonatais as infecções hospitalares são freqüentes devido à fragilidade dos mecanismos de defesa dos recém nascidos, que muitas vezes podem ser agravadas por complicações decorrentes da prematuridade e do baixo-peso aliadas à 
necessidade de procedimentos invasivos e a baixa adesão dos profissionais à higienização das mãos ${ }^{(20)}$.

As mudanças de comportamentos devem ser iniciadas com um processo de conscientização, de maneira singular, nas unidades cujos pacientes encontram-se sob a assistência direta dos profissionais de saúde durante a internação, ou submetidos a procedimentos invasivos, com solução de continuidade e dependência total ou parcial desses cuidados, dentre outros, pois, a tão almejada qualidade da assistência à saúde, somente será alcançada com o desenvolvimento de um processo educativo contínuo que mobilize os profissionais a se voltarem para essa temática, objetivando aprimorar os recursos humanos.

Pesquisa que investigou o direcionamento e o foco das ações dos gerentes de enfermagem das Unidades de Internação evidenciou que o enfermeiro ainda executa mais do que planeja ${ }^{(20)}$. O enfermeiro é considerado um elemento essencial nos serviços de saúde por ter a capacidade de articular as diversas ações propostas pela equipe multidisciplinar, devendo assim estar diretamente envolvido no planejamento de programas de educação permanente nas instituições de saúde.

\section{CONCLUSÕES}

No presente estudo foi identificado cocos gram positivos, bastonetes gram negativos, leveduras e fungos filamentosos nas mãos das mães, dos profissionais e de acadêmicos da área da saúde, os quais são apontados na literatura como frequentemente associados a surtos de infecção hospitalar em berçários e alojamentos conjuntos.

Há necessidade de operacionalização de programas educativos sobre higienização das mãos, dirigidos não só aos profissionais da área de saúde, mas também aos graduandos e às mães de recémnascidos. Destaca-se a importância desta temática ser introduzida e discutida já nos cursos de graduação e durante o seguimento pré-natal.

Por ser uma das medidas mais importantes de prevenção e controle de infecção hospitalar sua operacionalização exige articulação das instituições de saúde, educadores, profissionais, pacientes e cuidadores.

\section{REFERÊNCI AS}

1. Agência Nacional de Vigilância Sanitária. Higienização das mãos em serviços de saúde. Brasília: Anvisa; 2007.

2. Widmer AF, Conzelmann $M$, Tomic $M$, Frei $R$, Stranden AM. Introducing alcohol-based hand rub for hand hygiene: the critical need for training. Infect Control Hosp Epidemiol. 2007;28(1):50-4.

3. Cerqueira MCM. Antissepsia - Princípios Gerais e Antissépticos. In: Rodrigues EAC, Mendonça JS,
Amarante JMB, Alves Filho MB, Grinbaum RS, Richtmann R. Infecções Hospitalares Prevenção e Controle. São Paulo: Sarvier; 1997. p. 426-34.

4. Pimenta FC, Ito IY, Lima SNM. Biossegurança em Endodontia. In: Estrela C, Figueiredo JAP. Endodontia: Princípios Biológicos e Mecânicos. Säo Paulo: Artes Médicas; 1999. p. 412-64.

5. Ricardo SB. Emergência de S. Aureus MeticilinaResistente (MRSA) na Comunidade. Revista Prática Hospitalar. 2004;34:131-4.

6. Kuehnert MJ, Kruszon-Moran D, Hill HA, Mcquillan G, McAllister SK, Fosheim G et al. Prevalence of Staphylococcus aureus nasal colonization in the United States, 2001-2002. J Infect Dis. 2006;193(2):172-9.

7. Costa LS, Ribas RM, Beicher $A M A H$, Gontijo Filho PP. Presença de "Staphylococcus aureus" e bactérias Gram negativas nas mãos de profissionais de enfermagem, estudantes de medicina/médicos, e visitantes em diferentes unidades do Hospital de Clínicas da Universidade Federal de Uberlândia (HCUFU). In: Anais do $8^{\circ}$ Congresso Brasileiro de Controle de Infecção e Epidemiologia Hospitalar; 2002; Curitiba, Brasil. 2002. p. 238.

8. Gontijo Filho F, Rabelo N. Prevalência de IH e de colonização por Staphylococcus aureus e enterobactérias resistentes no berçário de alto risco do HC-UFU, MG. In: Anais do $6^{\circ}$ Congresso Brasileiro de Infecção Hospitalar; 1998 dez 30-03; Campos do Jordão, Brasil. 1998. p. 41.

9. Veiga F. Agentes de Infecção Hospitalar em UTI neonatal. In: Anais do VI Congresso Brasileiro de Infecção Hospitalar; 1998 dez 30-03; Campos do Jordão, Brasil. 1998. p. 73.

10. Jang TN, Fung CP, Yang TL, Shen SH, Huang CS, Lee $\mathrm{SH}$. Use of pulsed-field gel electrophoresis to investigate an outbreak of Serratia marcescens infection in a neonatal intensive care unit. J Hosp Infect. $2000 ; 48(1): 13-9$.

11. Becks VE, Lorenzoni NM. Pseudomonas aeruginosa outbreak in a neonatal intensive care unit: a possible link to contaminated hand lotion. Am J Infect Control. 1995;23(6):396-8.

12. Markovic-Denic L, Durisic J, Nikolic T, Ramadani $\mathrm{R}$, Ilic S, Stevanovic S. Causative agents of neonatal nosocomial infections and their resistance to antibiotics. Med Pregl. 2006;59(3-4):155-9.

13. Manzoni $P$, Farina $D$, Leonessa $M$, D'oulx EA, Galletto $P$, Mostert $M$ et al. Risk factors for progression to invasive fungal infection in preterm neonates with fungal colonization. Pediatrics. 2006;118(6):2359-64.

14. Whitby $M$, Pessoa-Silva $C L$, Mclaws $M L$, Allegranzi $B$, Sax H, Larson E et al. Behavioural considerations for hand hygiene practices: the basic building blocks. J Hosp Infect. 2007;5(1):1-8. 
15. Prado MA, Pimenta FC, Hayashida M, Souza PR, Pereira MS, Gir E. Enterobactérias isoladas de baratas (Periplaneta americana) capturadas em um hospital brasileiro. Am J Public Health. 2002;11(2):93-7.

16. Coia JE, Duckworth GJ, Edwards DI, Farrington M, Fry C, Humpreys $H$, Mallaghan $C$ et al. Guidelines for the control and prevention of meticillin-resistant Staphylococcus aureus (MRSA) in healthcare facilities. J Hosp Infection. 2006;63(Suppl 1):S1-44.

17. Karabey S, Ay P, Derbentli S, Nakipoglu Y, Esen F. Handwhashing frequencies in an intensive care unit. J Hosp Infect. 2002;50(1):36-41.

18. Akyol A, Ulusoy $H$, Ozen I. Handwashing: a simple, economical and effective method for preventing nosocomial infections in intensive care units. J Hosp Infect. 2006;62(4):395-405.

19. Pedigone MAMC, Dantas RHEA, Pereira JLS, Ribeiro MCFN, Huang WL. Comportamento Epidemiológico das Infecções Hospitalares (IH) no CTI Neonatal da Santa Casa de Franca de Abril/1998 à julho/2000. In: Anais do III Congresso PanAmericano, VII Congresso Brasileiro, I Congresso da Odontologia; 2000 Nov 10-14; Minas Gerais, Brasil. 2000. p. 97.

20. Coelho MA, Barbosa MA, Silva MML. Análise dos registros e se ações planejadas/executadas por gerentes de enfermagem de um hospital público. Rev. Eletr. Enf. [Internet]. 2008 [cited 2009 jul 30];10(3):573-9. Available from: http://www.fen.ufg.br/revista/v10/n3/v10n3a03.htm

Artigo recebido em 24.03.08.

Aprovado para publicação em 06.05.09.

Artigo publicado em 30.09.09. 